Cahiers $d u$ MONDE RUSSE

\section{Cahiers du monde russe}

Russie - Empire russe - Union soviétique et États indépendants

$43 / 4 \mid 2002$

Intellectuels et intelligentsia

\title{
L'intelligentsia et son rôle dans les organisations publiques de la ville de Tambov au tournant du XXe siècle
}

Essai d'étude régionale

Anastasija S. TUMANOVA

\section{OpenEdition}

\section{Journals}

Édition électronique

URL : https://journals.openedition.org/monderusse/8524

DOI : $10.4000 /$ monderusse. 8524

ISSN : $1777-5388$

Éditeur

Éditions de l'EHESS

Édition imprimée

Date de publication : 30 décembre 2002

Pagination : 645-660

ISBN : 2-7132-1796-2

ISSN : $1252-6576$

Référence électronique

Anastasija S. TUMANOVA, «L'intelligentsia et son rôle dans les organisations publiques de la ville de Tambov au tournant du XXe siècle », Cahiers du monde russe [En ligne], 43/4 | 2002, mis en ligne le 01 janvier 2007, consulté le 02 septembre 2022. URL : http://journals.openedition.org/monderusse/8524 ; DOI : https://doi.org/10.4000/monderusse.8524 
chercher : repérer : avancer

Cet article est disponible en ligne à l'adresse :

http://www.cairn.info/article.php?ID REVUE=CMR\&ID NUMPUBLIE=CMR 434\&ID ARTICLE=CMR 4340645

L'intelligent sia et son rôle dans les organisations publiques de la ville de Tambov au tournant du XXe siècle. Essai d'étude régionale

par Anast asija S. TUMANOVA

| Editions de l'EHESS | Cahiers du monde russe

2002/4 - Vol 43

ISSN 1252-6576 | ISBN 2713217962 | pages 645 à 660

Pour citer cet article :

-TUMANOVA A., L'intelligent sia et son rôle dans les organisations publiques de la ville de Tambov au tournant du XXe siècle. Essai d'étude régionale, Cahiers du monde russe 2002/ 4, Vol 43, p. 645-660.

Distribution électronique Cairn pour les Editions de l'EHESS.

(C) Editions de l'EHESS. Tous droits réservés pour tous pays.

La reproduction ou représentation de cet article, notamment par photocopie, n'est autorisée que dans les limites des conditions générales d'utilisation du site ou, le cas échéant, des conditions générales de la licence souscrite par votre établissement. Toute autre reproduction ou représentation, en tout ou partie, sous quelque forme et de quelque manière que ce soit, est interdite sauf accord préalable et écrit de l'éditeur, en dehors des cas prévus par la législation en vigueur en France. Il est précisé que son stockage dans une base de données est également interdit. 


\title{
L'INTELLIGENTSIA ET SON RÔLE DANS LES ORGANISATIONS PUBLIQUES DE LA VILLE DE TAMBOV AU TOURNANT DU XX ${ }^{\mathrm{e}}$ SIÈCLE
}

\author{
Essai d'étude régionale
}

Un trait caractéristique de l'intelligentsia russe a été son engagement important dans l'activité publique. Elle était l'inspirateur idéologique d'un grand nombre de mouvements sociaux, de partis politiques et fixait les orientations du développement culturel du pays. L'histoire de l'intelligentsia a été aussi marquée par sa contribution à la formation et à l'activité d'organisations publiques officielles; ces organisations bénévoles, regroupées en associations autonomes, firent leur apparition en grand nombre en Russie à la fin du XIX et au début du XXe siècle. Les auteurs prérévolutionnaires les appelaient des «sociétés d'initiative privée », soulignant ainsi leur caractère informel et l'orientation de leur action autour de la satisfaction des intérêts et des besoins privés.

Ces sociétés empiétaient sur des sphères comme l'éducation, la santé, la sécurité sociale, suppléant de manière sensible les actions du gouvernement et des organes locaux. L'étude des expériences et des traditions de l'initiative privée en matière sociale, ainsi que du rôle qu'y a tenu l'intelligentsia, permet de montrer la spécificité et les contradictions du processus de formation des institutions de la société civile en Russie.

Pour notre étude nous avons pris pour modèle Tambov d'avant la Révolution, un chef-lieu de province (gubernija) du centre de la Russie. Aussi bien son aspect extérieur que l'importance numérique et la composition de sa population ${ }^{1}$ en faisaient

1. La population de Tambov atteignait 50000 habitants au début du Xx siècle ; les paysans prédominaient, ils représentaient $49,7 \%$ de l'ensemble de la population, la noblesse $11 \%$, la bourgeoisie 29 \%. Pervaja vseobščaja perepis' naselenija Rossijskoj imperii 1897 g. (Premier recensement général de la population de l'empire russe, 1897), t. 42 : Tambovskaja gubernija, Saint-Pétersbourg, 1904, p. 54-55. 
un chef-lieu de province typique. Le mode de vie provincial marquait de son empreinte la définition et l'orientation des intérêts, le niveau culturel et l'organisation des loisirs des citoyens.

La vie publique d'une ville de province était, certes, moins intense que celle de la capitale; pourtant, l'étude de Tambov au tournant du siècle récuse l'image stéréotypée, véhiculée par la littérature, d'une société dont les valeurs matérielles et morales étaient peu élevées et peu propices au développement d'une vie culturelle comparable à celle qui s'observait au niveau national. En fait, la réalité socioculturelle de la province et celle de la capitale se distinguaient peu : les initiatives privées dans le secteur social suivaient des évolutions analogues, un intense travail intellectuel et spirituel était mené de part et d'autre, produisant les conditions nécessaires à l'éducation, la science et l'art.

Commencée à l'époque des « grandes réformes » dans les années 1860-1870, la modernisation sociale a touché la ville provinciale de Tambov, atténuant son retard par rapport aux autres centres culturels du pays. Toutes les sphères de la vie locale virent apparaître de nouvelles tendances et de nouvelles mentalités. Cet éveil fut perceptible avant tout dans l'éducation. Si, en 1894, on dénombrait 37 écoles dans la ville, il y en avait 46 en 1913, et on projetait d'en ouvrir encore sept ${ }^{2}$. La presse témoigna de l'intérêt grandissant de la société pour l'éducation, elle se fit alors spontanément l'écho des changements, sensible à la dimension critique de cette époque de transition. «Le temps du silence séculaire, là-bas, dans les profondeurs de la Russie, est révolu ; on y exige désormais des connaissances solides $»^{3}$, écrivait le journal Tambovskie otkliki. «L'aspiration générale à la culture a touché même notre petite province [...]. Le livre, la revue et le journal sont entrés peu à peu dans les mœurs ordinaires $»^{4}$, lui faisait écho Tambovskaja žizn'.

Au début du siècle à Tambov paraissaient près de quinze périodiques de tendances idéologiques et politiques diverses. C'étaient surtout des publications privées qui, plus que les publications officielles, satisfaisaient généralement l'intérêt du lecteur en lui offrant une description vivante de la vie de la province dans ses multiples aspects. Celle-ci s'enrichissait aussi de nouvelles formes d'activités culturelles et éducatives, inconnues auparavant, comme les conférences publiques, les soirées littéraires et musicales. Les attentes intellectuelles sans cesse grandissantes des habitants étaient satisfaites par les bibliothèques et les salles de lecture. À en juger par les demandes des lecteurs, les goûts littéraires du public de Tambov étaient assez hétérogènes, privilégiant les œuvres classiques de la littérature russe : Tolstoj, Čehov, Turgenev, Dostoevskij. Les publications périodiques de la capitale étaient connues : Vestnik vospitanija, Russkoe bogatstvo, Sovremennik 5 . Dans les années 1870, Tambov

2. «Narodnoe prosveščenie » (L'instruction publique), in Pamjatnaja knižka Tambovskoj gubernii na 1894 g. (Agenda de la gubernija de Tambov pour 1894), Tambov, 1894, p. 332 ; Tambovskij kraj, 6 décembre 1913.

3. Tambovskie otkliki, 16 janvier 1914.

4. Tambovskaja žizn', 11 juin 1914.

5. Archives d'État de l'oblast' de Tambov (Gosudarstvennyj arhiv Tambovskoj oblasti GATO), f. 25, op. 1, d. 27, 1.27, 80 v., 129, 165, 284, 286 : Tambovskie otkliki, 3 mai 1914. 
occupait une des premières places parmi les villes de province, sous le rapport du nombre d'abonnés au Vestnik Evropy, journal historico-politique lu dans les milieux de l'intelligentsia libérale 6 .

La culture artistique connut de réels changements. Le cinéma fit son apparition ainsi que des ateliers de théâtre et d'arts plastiques, des expositions furent organisées, ainsi que des concerts symphoniques, des spectacles d'opéra et de théâtre par des professionnels, privilège jusqu'alors réservé aux seuls habitants des capitales. La grande comédienne du Malyj Teatr, G. N. Fedotova, et le comédien du MHAT, V. I. Kačalov, vinrent en tournée au théâtre de Tambov, dans lequel I. M. Moskvin, entre autres, fit ses débuts.

Le développement des moyens de communication a joué aussi son rôle dans l'évolution de la qualité de la vie. La construction d'un embranchement ferroviaire desservant le chef-lieu de district Kozlov mit fin à l'isolement séculaire de la province : les événements culturels mettaient moins de temps à y parvenir et les nouvelles circulaient plus vite entre Tambov et les autres villes de l'empire. La participation à des cérémonies commémoratives importantes, tels le centenaire de la guerre de 1812, le tricentenaire de la dynastie des Romanov ou le cinquantenaire de la création des zemstva, favorisa l'intégration de la province à l'environnement culturel russe.

La participation aux campagnes électorales et au travail courant du self-government local permit aux habitants de Tambov d'élargir leur horizon politique et de se familiariser avec l'action concrète. Les élections à la douma municipale, avec leurs nombreuses réunions préélectorales et leurs discussions à propos des listes de candidats de l'alternance, eurent un grand retentissement dans le public.

Le développement de l'action publique à Tambov au tournant du $\mathrm{Xx}^{\mathrm{e}}$ siècle entraîna nécessairement un changement dans l'attitude des citadins vis-à-vis de l'action publique et une plus grande conscience de soi et de l'action citoyenne. «L'habitant de Tambov a fait un grand pas en avant vers une plus grande conscience de son rôle public, un plus grand intérêt pour ce qui [...] sort du cadre de la vie personnelle. Le petit-bourgeois laisse la place à la société... $\gg^{7}$, note le journaliste social-démocrate V.N. Podbel'skij. L'expérience de l'administration publique et culturelle brisa peu à peu l'isolement de la ville du reste du monde, l'intégrant harmonieusement au système général des échanges culturels.

L'intelligentsia joua un rôle fondamental dans la vie publique de Tambov ${ }^{8}$. L'abolition du servage, les progrès dans le domaine social et le développement économique rapide du pays au tournant du $\mathrm{XX}^{\mathrm{e}}$ siècle rendirent nécessaire l'éléva-

6. V. N. Kozljakov, A. A. Sevast'janova, «Kul'turnaja sreda provincial’nogo goroda» (Le milieu culturel de la ville de province), in Očerki russkoj kul'tury XIX veka (Études de la culture russe du XIX $X^{e}$ siècle), t. 1, Moscou, 1998, cf. le tableau p. 179-180.

7. Tambovskaja žizn',26 mai 1913.

8. Pour l'auteur, l'intelligentsia comprend les personnes exerçant dans leur profession une activité intellectuelle dans des branches diverses : les enseignants, le personnel médical, les représentants des professions libérales, le clergé, les ingénieurs, les avocats, les fonctionnaires, les étudiants. 
tion du niveau culturel et éducatif de la société russe et élargirent la sphère d'activité des intellectuels. Ceci eut pour effet le gonflement du nombre d'intellectuels de la classe moyenne dans les villes. À Tambov, entre 1897 et 1917, leurs effectifs passent de 1054 à 2041 (dont une augmentation de 466 à 748 dans les milieux éducatif et culturel, et de 420 à 502 dans le personnel médical) ${ }^{9}$.

Rappelons ici que l'aspiration de l'intelligentsia russe à servir son peuple avec abnégation a toujours été un trait essentiel caractéristique de ses meilleurs éléments. Le besoin de se réunir pour débattre des thèmes sensibles de la vie publique ou pour organiser des loisirs collectifs renvoie aussi aux particularités du caractère national russe où dominent la sociabilité et l'ouverture intellectuelle et émotionnelle à l'entourage. L'historien contemporain B. F. Egorov, qui a étudié la mentalité de l'intellectuel russe de cette époque, voit en ce dernier « moins de pragmatisme et d'intérêt personnel que chez son homologue occidental et une plus grande prégnance de cette aspiration romantique à débattre de la culture et de tous les problèmes du monde ou des moyens à mettre en œuvre pour transformer la mère-patrie. » ${ }^{10}$

Groupe social n'appartenant à aucune classe sociale en particulier et aspirant à être l'interprète des intérêts et des attentes des diverses catégories de la population, l'intelligentsia se distinguait par la grande variété de ses activités et de ses orientations. À Tambov, la « classe instruite » s'occupait des questions les plus diverses, allant de l'organisation des services municipaux au développement de l'éducation, de la santé publique et de la culture. La participation des différents groupes d'intellectuels aux associations publiques de Tambov dépendait de toute une série de facteurs d'ordre économique, socio-culturel et psychologique, telles l'aisance matérielle, l'éducation ou la classe sociale. Ainsi l'influence des enseignants futelle le plus marquée dans les organisations à mission pédagogique, dans les sociétés professionnelles d'entraide aux enseignants et dans les sociétés de recherche ethnographique locale. Les créateurs artistiques se regroupaient principalement dans les sociétés de type culturel et récréatif.

Il se dégage trois groupes principaux parmi les organisations publiques qui regroupaient l'intelligentsia de Tambov: pédagogiques, savantes, et de type récréatif orientées vers l'art et la culture. Elles œuvraient toutes dans le domaine socio-culturel, domaine au sein duquel les aptitudes et les aspirations des milieux cultivés s'accordaient de façon organique avec les exigences et les besoins sociaux concrets de la population de Tambov. Le secteur éducatif visant à la formation scientifique et artistique de la population favorisa, au sein de l'intelligentsia, le développement des moyens d'expression et de la compétence professionnelle. Dans une ville de province, dont la vie sociale n'était pas particulièrement trépi-

9. V. V. Kaniščev, « Tambovskaja intelligencija v XIX - načale XX vv. » (L'intelligentsia de Tambov au XIX et au début du XX ${ }^{\mathrm{e}}$ siècle), in Očerki istorii kul'tury Tambovskogo kraja. Sbornik naučnyh rabot (Essais d'histoire culturelle du kraj de Tambov. Recueil de travaux scientifiques), Tambov, 1993, p. 54.

10. B. F. Egorov, « Russkie kružki » (Les cercles russes), in Iz istorii russkoj kul'tury (De l'histoire de la culture russe), t. 5 : XIX vek (XIXe siècle), Moscou, 1996, p. 507. 
dante, la mission culturelle de l'intelligentsia prenait un poids et une actualité particulière.

L'intelligentsia de Tambov se mobilisa tout d'abord sur le développement de l'éducation, ce qui se justifiait par la situation délicate dans ce secteur. Ainsi, au tournant du XX $\mathrm{XX}^{\mathrm{e}}$ siècle, l'indice d'alphabétisation dans la province de Tambov atteignait en tout $16,6 \%$ (25\% chez les hommes et $7 \%$ chez les femmes); à Tambov même, le nombre de personnes sachant lire et écrire représentait $54 \%$ de la population ${ }^{11}$. De sérieuses carences pouvaient s'observer dans l'organisation de l'enseignement scolaire : le manque de moyens matériels, de locaux et d'encadrement pédagogique compétent était flagrant.

L'éventail des activités pédagogiques de l'intelligentsia de Tambov était assez large : l'attention se portait en grande partie sur le développement de l'enseignement primaire et secondaire et sur l'instruction religieuse et morale. Mais c'est dans le domaine de la formation extrascolaire des enfants et des adultes - une branche de l'éducation à laquelle ni le gouvernement ni les organes locaux du pouvoir ne s'intéressaient suffisamment - que l'on obtint les meilleurs résultats ${ }^{12}$.

C'est la Société de conférences publiques (Obščestvo narodnyh čtenij), ouverte en 1893, qui devint le centre de formation extrascolaire de Tambov. Les statuts de cette société furent rédigés spécialement pour la province de Tambov car il n'existait pas encore d'organisations de ce type en Russie. Les conférences publiques étaient l'initiative de M. T. Popov, professeur de lycée classique. Les enseignants du lycée et de l'école professionnelle formaient le noyau de l'organisation. Le soutien financier était assuré par E. D. Naryškin, grand propriétaire foncier de Tambov et mécène renommé. Ses dons permirent de construire un bâtiment de deux étages dans lequel on installa une bibliothèque, une salle de lecture, un musée historico-ethnographique et une grande salle de 600 places pour les conférences publiques.

À Tambov et dans les villes et villages de la province, les conférences avaient lieu huit mois sur 12, de septembre à mai, les dimanches et jours fériés. Le nombre moyen annuel de conférences atteignait presque les 90, avec un nombre d'auditeurs se situant entre 40000 et 50000 (entre 400 et un peu plus de 500 personnes par séance). En 1902, il y eut des conférences dans 294 localités de la province ${ }^{13}$ : ces chiffres permettent de se rendre compte de l'importance de l'activité de la société et de la popularité de ses manifestations auprès de la population. Vers 1909, la salle de

11. Pervaja vseobščaja perepis’́..., op. cit., p. XIII, 1, 56-57.

12. En 1914, les dépenses du zemstvo de Tambov relatives à l'éducation extrascolaire représentaient au total $1 \%$ de ses dépenses pour l'éducation: «Zadači zemstva v oblasti vneškol'nogo obrazovanija » (Les objectifs du zemstvo dans le domaine de l'éducation extrascolaire), Tambovskij kraj, 25 mars 1914.

13. « Očerk desjatiletnej dejatel’nosti Obščestva po ustrojstvu narodnyh čtenij v g. Tambove i Tambovskoj gubernii v svjazi s istoriej ego obrazovanija » (Aperçu de dix ans d'activité de la Société de conférences publiques dans la ville et la province de Tambov et histoire de sa formation), Sbornik-kalendar' Tambovskoj gubernii na 1903 god (Almanach de la province de Tambov pour l'année 1903), Tambov, 1903, p. 225. 
lecture de Tambov comptait déjà 14 sections réparties dans les écoles, les hôpitaux et les unités militaires de la ville ${ }^{14}$.

La population était informée par voie d'affiche de l'heure et du programme des conférences. Afin de donner plus d'attrait et de variété à celles-ci, on y invitait un chœur populaire et la fanfare militaire. En fait, ces manifestations tendirent à prendre la forme de spectacles dès 1913, lorsqu'il fut décidé de les accompagner d'une projection de film. C'étaient des membres de la société - en grande partie des enseignants des écoles de la ville - qui étaient responsables des conférences. Les thèmes des conférences et des débats étaient des plus variés, touchant la littérature, l'histoire ou la religion. La programmation annuelle tenait compte des dates marquantes du calendrier culturel russe. La conférence donnée à l'occasion du tricentenaire du règne de la dynastie des Romanov, qui rassembla plus de 1200 personnes, laissa aux habitants de Tambov un souvenir inoubliable ${ }^{15}$.

Devant le succès des conférences, les dirigeants de la société eurent l'idée d'améliorer la qualité de leur travail sous toutes ses formes. L'étape suivante fut la construction de bibliothèques et de salles de lecture publiques. La direction de la société recevait un grand nombre de lettres demandant de l'aide pour l'ouverture de bibliothèques dans les villages. Entre 1895 et 1903, il s'ouvrit en tout 725 bibliothèques publiques dans la province, alors que leur nombre avant 1895 ne dépassait même pas la dizaine. En créant ces bibliothèques et en les équipant, non seulement la société apprenait à connaître les goûts de la population en matière de lecture mais elle essayait aussi de les influencer et de donner au public le goût des lectures sérieuses.

Grâce à la diversité de son activité culturelle et pédagogique, la Société de conférences publiques vit sa popularité augmenter dans la population et reçut dans ses rangs des collaborateurs qualifiés et capables d'une grande initiative. Vers 1915, elle comptait déjà 232 membres $^{16}$. Sa composition professionnelle en faisait une organisation typiquement intellectuelle, le plus gros de ses troupes étant formé d'enseignants (47\% des membres selon les chiffres de 1915), de membres du clergé (27\% en 1915), de médecins, d'avocats et de personnalités du zemstvo ${ }^{17}$.

Pour ce qui est de l'appartenance politique de ses membres, la Société de conférences publiques présentait une assez grande variété. On y trouvait des représentants de partis et de mouvements politiques divers et variés, allant de l'extrême droite aux socialistes révolutionnaires (SR): des monarchistes (M. T. Popov, A. A. Ščegolev), des nationalistes (S. O. Šadrov), des progressistes (S. I. Komsin), des membres du parti constitutionnel-démocrate (cadets) (L. D. Brjuhatov, N. N. Satin, A. Ja. Timofeev) et des SR (K. N. Šatov). Les idées des Lumières,

14. GATO, f. 25, op. 1, d. 27,1. 102.

15. Otčet pravlenija Ob̌̌čestva po ustrojstvu narodnyh čtenij $v g$. Tambove i Tambovskoj gubernii za 1913 god (Compte rendu du conseil d'administration de la Société de conférences publiques dans la ville et la province de Tambov pour l'année 1913), Tambov, 1915, p. 14.

16. GATO, f. 25, op. 1, d. 142,1.126.

17. Établi d'après GATO, f. 25, op. 1, d. 142,1. 126. 
populaires au sein des milieux cultivés de la ville, rassemblaient des personnalités publiques de tout bord qui oubliaient alors leurs divergences politiques fondamentales pour vivre en bonne intelligence dans le cadre d'une même organisation ou au sein du même conseil d'administration.

Cependant, les intérêts de parti ne furent pas sans affecter les relations des membres de la Société de conférences publiques à certaines périodes spécifiques de son existence. Ainsi, pendant la révolution de 1905-1907, le conseil d'administration de la Société, qui était d'orientation progressiste, mit à la retraite son président, L. I. Uspenskij, directeur du lycée de la province, homme de droite. Le conseil était composé de L. D. Brjuhatov, membre du conseil du zemstvo de la province; A. Ja. Timofeev, avocat; T.E. Ostroumov, inspecteur des écoles publiques; I. I. Zolotouhin, membre de la douma municipale. À cette époque, la salle de lecture publique se transforma en centre de réunions et de meetings agités, et de discussions sur des questions politiques d'actualité. La société ne retrouva son ancien loyalisme que grâce aux efforts « hérö̈ques » du gouverneur N. P. Muratov, qui déploya tout l'arsenal des mesures administratives dont il disposait. Deux exemples parmi d'autres: Brjuhatov fut arrêté et Ostroumov fut chassé de la province ${ }^{18}$.

La Société de conférences publiques établit des bases solides pour le développement de l'éducation extrascolaire à Tambov. Vingt ans plus tard, en 1912, l'entreprise des pédagogues de Tambov jouissait du soutien des juristes qui avaient fondé une organisation dont la mission était similaire, la Société de la bibliothèque (Ob̌̌čestvo biblioteki). Pendant toute cette période, le nombre maximal des membres de cette société oscilla entre 50 et 60 personnes, pour la plupart issues des secteurs juridique et pédagogique, et de l'administration des zemstva. La société avait à sa tête le prince Išeev, personnalité publique bien connue à Tambov, qui était aussi l'un des dirigeants de la section locale du parti constitutionnel-démocrate. Les membres actifs de cette jeune organisation étaient d'autres cadets, les avocats N. M. Nazar'ev, I. N. Spasskij et M. K. Vol'skij. Dès sa création, la société fut l'objet d'une surveillance sans relâche de la part de la direction locale de la gendarmerie. Dans une lettre secrète du 8 décembre 1912 adressée à son chef V. M. Fon-Oglio, le gouverneur de Tambov, N. F. Ošanin, fait part de ses doutes sur la loyauté politique de la société, dont la majorité des fondateurs appartenait au parti constitutionnel-démocrate et avait fait preuve d'une grande activité aux élections de la IVe douma d'État. La société ne serait-elle pas un simple écran utilisé par l'association des cadets de Tambov pour bénéficier du droit légal de réunion? se demandait le gouverneur ${ }^{19}$. Il ordonna qu'on surveille attentivement la société, mais les craintes des autorités s'avérèrent sans fondement. La société correspondait bien à son intitulé et s'occupait strictement de l'accomplissement de sa mission pédagogique.

18. GATO, f. 25, op. 1, d. 142,1.90 vo-91.

19. GATO, f. 272 , op. 1, d. $9,1.23 \mathrm{v}^{\circ}$. 
Une bibliothèque et une salle de lecture furent créées grâce aux efforts des membres de la société. En 1916, la bibliothèque comptait déjà 10500 volumes, et le nombre de ses visiteurs atteignait presque les 2000 personnes ${ }^{20}$. Contrairement aux bibliothèques de la Société de conférences publiques, cette bibliothèque s'adressait principalement à un public cultivé. Les personnes ayant fait des études supérieures ou secondaires, y compris les élèves d'établissements secondaires spécialisés, représentaient entre 70 et $80 \%$ de ses visiteurs ${ }^{21}$. Diverses personnalités se rendirent à l'invitation de la Société de la bibliothèque pour donner des conférences : l'écrivain F. K. Sologub, le critique d'art S. Glagol', les critiques littéraires moscovites Ju. I Ajhenval'd et N. Ju. Abramovič.

Au cours de la décennie qui s'étendit entre les deux révolutions (1907-1916), l'éventail des activités des organisations pédagogiques de Tambov s'enrichit de nouvelles formes de travail sortant de la tradition: la Société d'enseignement secondaire (Obščestvo srednego obrazovanija) (1916) créa un gymnase de garçons qui offrait un soutien matériel aux lycéens nécessiteux, tandis que le club des enseignants - le Cercle pédagogique (Pedagogičeskoe sobranie) - fut l'initiateur en 1910 de tribunaux d'honneur destinés à juger les héros de littérature populaire ; ces tribunaux commencèrent à fonctionner dès 1913.

Parmi les sociétés publiques de l'intelligentsia de Tambov, on trouvait un groupe particulier consacré à l'art et aux loisirs culturels. Au départ, les conditions de développement de ce type d'activité à Tambov n'étaient pas particulièrement favorables. À la fin du XIX ${ }^{\mathrm{e}}$ siècle, la ville comptait encore très peu d'organisations à vocation culturelle et pédagogique, et il y avait encore peu de personnes sensibilisées à l'art. D'où le caractère informel des premières associations d'amateurs d'art. On trouvait des clubs de dessin et de photographie, des cercles privés qui se réunissaient occasionnellement et qui regroupaient l'élite de la ville. Cependant, au fur et à mesure que l'intérêt de la société pour la création artistique grandissait, l'intelligentsia commença à envisager la création d'associations à la structure définie et fixe comportant un programme d'activités permanentes.

L'ouverture en 1882 d'une section de la Société russe de musique (Russkoe muzykal'noe obščestvo - RMO) marqua une étape décisive dans le développement de la culture musicale de la province de Tambov. La RMO ouvrit pour toute la Russie prérévolutionnaire près de 50 de ces filiales. La direction de la section de Tambov fut confiée à un collectif composé de N. N. Čolokaev, maréchal de province de la noblessse, V. M. Petrovo-Solovovo et K. A. Benkendorf, maréchaux de district de la noblesse, I. A. Guadanini, le maire, et de plusieurs autres responsables du self-government local.

Dans le cadre de la société on donnait des cours de musique qui finirent par se transformer en école - un établissement de niveau plus élevé qui préparait au

20. Otčet Obščestva Tambovskoj biblioteki za 1916 god (Rapport de la Société de la bibliothèque de Tambov pour l'année 1916), Tambov, 1917, p. 5, 11.

21. Compte établi d'après Otčet Obščestva Tambovskoj biblioteki za 1913 god (Rapport de la Société de la bibliothèque de Tambov pour l'année 1913), Tambov, 1914, p. 8, 9; Otčet Obščestva Tambovskoj biblioteki za 1916 god, p. 5, 6. 
certificat de fin d'études musicales secondaires. L'école fut ouverte en 1900. Trois ans plus tard, elle s'installa dans un bâtiment récemment construit à l'intersection de deux rues centrales de la ville, Bol’̌saja et Dvorjanskaja. Elle était dirigée par un ancien élève du Conservatoire de Moscou, S. M. Starikov, pianiste et chef d'orchestre de talent, qui s'était rendu à l'invitation de la section de la RMO de Tambov. Le nom de Starikov est indéfectiblement lié à l'école de musique : il fut à l'origine de sa création, il y mit au point les programmes d'études et y forma une génération entière de musiciens professionnels. Parallèlement, il monta le premier orchestre symphonique de la région, remit en activité une section de chant choral et œuvra efficacement en faveur de l'art vocal.

Des concerts symphoniques commencèrent à se mettre en place sous l'égide de l'école. Les chanteurs F. I. Šaljapin, L. V. Sobinov, A. V. Neždanova, A. D. Vjal'ceva, les pianistes A. Ziloti, A. Gol'denvejzer, E. Frej et bien d'autres encore vinrent en tournée à l'invitation de la section de la RMO de Tambov. Ils étaient acccompagnés de l'orchestre symphonique des élèves de l'école sous la direction de Starikov. Comme il y avait beaucoup de musiciens de talent parmi les premiers enseignants de l'école, le jeu des jeunes musiciens était d'un niveau plutôt élevé. La sélection des œuvres et les répétitions s'effectuaient au cours de réunions musicales en petit comité qui avaient lieu le jeudi dans les locaux de l'école. La société donnait plusieurs concerts de musique symphonique et de musique de chambre par saison, soumettant au jugement du public le jeu des jeunes musiciens ; ces soirées attiraient une assistance fidèle et empressée.

La société de musique traversa une série d'épreuves entre 1909 et 1912, lorsque le gouverneur de la province, N. M. Muratov, commença à mettre un frein à l'activité artistique de l'école. Son antisémitisme était à l'origine de ces agissements. De son propre aveu, pendant presque toute la durée de ses fonctions à ce poste, il avait « lutté contre la mainmise des juifs sur tous les secteurs de la vie de la province et ne pouvait naturellement pas fermer les yeux sur le fait que le représentant de la Société impériale russe de musique de la ville de Tambov était le mécréant juif Starikov ${ }^{22}$. L'école fut mise sous la surveillance acharnée du gouverneur qui l'observa sans relâche. Les concerts de l'orchestre symphonique de l'école dirigée par Starikov furent tacitement interdits et le chef d'orchestre ne fut plus invité aux soirées et aux réunions musicales solennelles auxquelles assistaient le gouverneur et la haute société de la ville. Cependant, les obstacles créés par l'administration ne parvinrent pas à réduire l'activité de la société. Au plus fort des persécutions, pendant la saison 1910-1911, la section de la RMO monta huit concerts, trois représentations d'élèves et un opéra, tous interprétés de façon magistrale ${ }^{23}$.

L'attitude du gouverneur envers la Société de musique suscita des réactions négatives dans l'opinion publique locale. A. N. Naryškina, dame d'honneur à la Cour et mécène renommé de la province, prit le parti du maestro disgracié, exigeant du gouverneur qu'il mît fin à la « campagne contre les pédagogues » de l'école et

22. GATO, f. 4, op. 1, d. 6848,1. 123.

23. Ibid., 1. 48-49. 
qu'il fît preuve de «plus de modération et de sang-froid». Le prince N. N. Čolokaev, maréchal de province de la noblesse, manifesta lui aussi son soutien à la société pendant cette période difficile de son existence. Alors que Muratov fermait les portes des institutions de la ville aux musiciens, Čolokaev mit la salle du Cercle de la noblesse (Dvorjanskoe sobranie) à la disposition de la société et devint l'auditeur principal de ses concerts. Il défendit souvent les intérêts de la société et de ses dirigeants auprès de la direction de Saint-Pétersbourg (étant donné que la princesse E. G. Al’tenburgskaja, présidente de la Société impériale russe de musique, apportait son appui à Starikov pour lequel elle avait beaucoup d'estime) et auprès du ministère des Affaires intérieures ${ }^{24}$.

Si Muratov ne parvint pas à obtenir la destitution de Starikov de la direction de l'École de musique, c'est en grande partie grâce à Čolokaev, doyen des personnalités du zemstvo, chef autoritaire et influent de la noblesse de Tambov, qui occupa ce poste sans interruption de 1891 à octobre 1917. En plus de ses activités au sein du self-government local, Čolokaev participait activement à des associations d'amateurs bénévoles : entre autres, la Société agricole (Sel'skohozjajstvennoe obščestvo), la Société de conférences publiques et la Société de protection des pauvres (Popečitel'noe obščestvo o bednyh). Il était aussi président du conseil d'administration des sections locales de la Société de musique et de la Société d'arboriculture fruitière et de culture maraîchère (Obščestvo plodovodstva $i$ ogorodničestva).

Dans la sphère de l'art figuratif, l'activité culturelle et pédagogique était placée sous la responsabilité de la Société des amateurs d'art (Obščestvo ljubitelej hudožestv) et de la Société de photographie (Fotografičeskoe obščestvo), toutes deux créées en 1906. Grâce aux efforts des militants de ces organisations, qui étaient principalement des professeurs de dessin des écoles de la ville, on organisa des expositions annuelles de peinture et de photographie où l'on pouvait voir les œuvres d'artistes célèbres - V. D. Polenov, A. M. Vasnecov, K. A.Korovin et d'autres et on installa des ateliers de peinture et des laboratoires de photographie.

Les associations de loisirs formaient un groupe important. Leur activité reflétait les centres d'intérêt propres à des groupes particuliers de la population qui y investissaient leur temps libre. Par exemple, la section locale de la Société pétersbourgeoise des loisirs pour enfants (S.-Peterburgskoe obščestvo detskih razvlečenij) organisait des activités pour les enfants, la Société des amateurs du Gramophone (1908) (Obščestvo ljubitelej grammofona) s'adressait plus particulièrement au personnel médical de l'hôpital du zemstvo de la province, qui s'intéressait fortement à cette nouvelle technique. La Société pour une bonne éducation physique (1909) (Obščestvo pravil'nogo fizičeskogo vospitanija) comptait une majorité de pédagogues et de membres du personnel médical que la nature même de leur activité incitait à attacher de l'importance à la culture physique et au sport.

24. Ibid., 1. 23-24, 123 ; Archives nationales de Russie de la littérature et des arts (Rossijskij gosudarstvennyj arhiv literatury i iskusstva - RGALI), f. 1208, op. 1, d. 26, 1. $156 \mathrm{v}^{\circ}, 176$. 
L'intelligentsia de Tambov joua un rôle décisif dans les sociétés savantes, dont l'importance était extrêmement grande dans la ville. Comme celle-ci n'avait ni établissement d'enseignement supérieur ni centre de recherche scientifique, les sociétés savantes étaient les seuls centres intellectuels qui se chargeaient de faire connaître la science. Le petit nombre de spécialistes parmi leurs membres poussa les habitants de Tambov à s'impliquer davantage dans ces activités et à accomplir tout un ensemble de missions : ils initièrent un large public à la science, contribuèrent à doter la ville de sa propre école de formation de chercheurs et tentèrent de réduire le fossé entre le niveau de développement scientifique de leur ville provinciale et celui des centres de recherche de la capitale.

Toutes les sociétés savantes se sont formées selon le même schéma, autour d'une ou de plusieurs personnalités hors du commun spécialisées dans une branche donnée de la science. Dans le cas de la Commission scientifique d'archives, c'étaient les ethnographes I. I. Dubasov et A. N. Norcov ; dans celui de la Société des amis de la nature (Obščestvo ljubitelej prirody), le géologue F. V. Lungersgauzen et le médecin V. N. Levčuk; dans celui de la Société d'histoire militaire (Voenno-istoričeskoe obščestvo), le lieutenant général et chef de garnison E.Z. Korbut ; et dans le cas de la Société physico-médicale (Fiziko-medicinskoe obščestvo), c'étaient le docteur en médecine E. H. Ikavitc et l'inspecteur médical de province V.K. Zedergol'm. La plupart d'entre eux avaient fait de l'histoire régionale leur centre d'intérêt. Ceci s'expliquait par le faible développement de cette branche ainsi que par le désir des ethnographes de Tambov de démentir l'opinion répandue selon laquelle l'histoire et la culture provinciales étaient des sujets ternes et sans avenir et de montrer le rôle unique et original de la province dans la vie du pays. Rappelons ici à propos la déclaration de l'historien-ethnographe Dubasov que «c'est précisément la province russe qui est l'essence de la vie en Russie ».

La société historique la plus ancienne de Tambov est la Commission scientifique d'archives (Tambovskaja učenaja arhivnaja Komissija - TUAK). Fondée en 1884 par ordre du tsar, elle fait partie des quatre premières commissions d'archives de province (Gubernskie učenye arhivnye Komissii - GUAK) ${ }^{25}$. Elle donnait à la fois l'image d'un organisme semi-étatique ayant pour mission de collecter les archives de la province et celle d'un organisme indépendant de recherche. Sa mission principale, en tant que société scientifique d'archives, était de collecter, de trier et de conserver les documents historiques. Cependant, la définition des priorités et de l'orientation de l'activité de la Commission dépendit beaucoup, au cours des différentes périodes de son existence, des goûts et des passions scientifiques de ses présidents. Il y en eut deux en tout, I. I. Dubasov et A. N. Norcov. Le premier, issu du clergé, historien et ethnographe, fut l'un des premiers à faire une étude systématique de l'histoire de la région de Tambov. Il laissa derrière lui un héritage scientifique considérable. Norcov, quant à lui, était un ancien militaire issu de la

25. Avant la révolution, il y avait en tout en Russie 39 commissions scientifiques d'archives de province. 
noblesse qui s'intéressa à la recherche lorsqu'il prit sa retraite. Comme il s'était toujours intéressé à l'action publique, il ne devint pas un savant de cabinet, et se fit connaître en tant que personnalité publique parmi les archivistes.

La première période de l'existence de la Commission, depuis sa création jusqu'à 1900, date à laquelle Dubasov quitta Tambov, fut consacrée à la collecte de matériaux documentaires et à la création d'un fonds d'archives historiques. Le mérite du premier directeur fut avant tout d'attirer des collaborateurs dynamiques et solides, dont une partie contribua à l'organisation des recherches scientifiques tandis que l'autre fournit le soutien matériel.

L'activité scientifique de la Commission s'organisa de telle façon qu'aucune réunion publique ne pouvait avoir lieu sans qu'il y eût un rapport scientifique ou une communication de son président. La Commission avait un très grand nombre de membres, près de 200 en 1904 et pas loin de 300 en 191726. Dans les listes des membres demeurant dans d'autres villes figurent des historiens et des archivistes renommés tels V. O. Ključevskij, I. E. Zabelin, D. Ja. Samokvasov, D. I. Ilovajskij, S. F. Platonov, S. M. Seredonin et A. S. Lappo-Danilevskij. Cependant, la recherche active était assurée par moins d'un dixième du groupe des collaborateurs, pour la plupart membres de l'intelligentsia locale.

L'activité de la Commission se limitait à deux grandes branches : l'activité interne, en rapport avec la mission de la Commission d'archives en tant que société savante et l'activité extérieure, orientée vers la vulgarisation scientifique et l'éducation de la population de Tambov. Le travail scientifique de la Commission consistait à collecter des documents historiques, à les conserver dans un dépôt unique et à les traiter. Les collaborateurs de la Commission réussirent à collecter près de 10000 dossiers dans ce dépôt. Le fonds historique contenait des documents uniques sur la colonisation des confins de l'ancienne Moscovie, une abondance de matériaux sur l'histoire de l'église et de la noblesse de Tambov et sur la vie quotidienne de la population de la ville. Dans l'ensemble, les intérêts scientifiques des membres de la Commission de cette période se limitaient à l'étude de l'histoire quotidienne de la population locale. Ceci était dû en grande partie aux centres d'intérêt de son président Dubasov qui pensait que « la vie quotidienne du peuple avec ses préoccupations journalières et ses innombrables centres d'intérêt, où qu'elle soit, dans le Centre, au fin fond de la province ou dans les confins, est le sujet de recherche historique le plus important $»^{27}$.

Comme les membres de la Commission ne voulaient pas se limiter à la seule collecte de documents d'archives, ils inclurent dans leurs occupations la recherche et la description d'objets historiques. Le musée de la Commission abritait une grande

26. «Kratkij istoričeskij očerk vozniknovenija i dejatel'nosti Tambovskoj učenoj arhivnoj komissii, 1884-1904 » (Bref aperçu historique de la création et de l'activité de la Commission scientifique d'archives de Tambov, 1884-1904), in Gubernskie učenye arhivnye komissii i ih značenie (Les commissions scientifiques d'archives de province et leur importance), Tambov, 1904, p. 9 ; Izvestija Tambovskoj učenoj arhivnoj komisssii (cité infra - ITUAK), 57, 1917.

27. I. I. Dubasov, Očerki istorii Tambovskogo kraja, (Essais d'histoire du kraj de Tambov), Moscou, 1883, fasc. 2, p. 1. 
variété de pièces d'archéologie, de minéralogie, de numismatique, d'antiquités religieuses et d'objets représentatifs du folklore local. Au début du XX $\mathrm{XX}^{\mathrm{e}}$ siècle, le nombre moyen de ses visiteurs se situait entre 30 et 50 personnes par jour ${ }^{28}$. La Commission se mit en contact permanent avec un grand nombre d'institutions scientifiques, dont la Société historique russe (Russkoe istoričeskoe obščestvo) et la Société d'archéologie de Moscou (Moskovskoe arheologičeskoe ob̌̌čestvo), la Société d'archéologie, d'histoire et d'ethnographie de Kazan (Kazanskoe obščestvo arheologii, istorii i etnografii), dans le but d'échanger des informations et de compléter les fonds de ses archives, de son musée et de sa bibliothèque.

L'activité culturelle et pédagogique de la Commission visait à propager la connaissance de l'histoire dans la population et à éveiller en elle un intérêt pour son passé. Pendant la durée de son existence, elle publia 74 ouvrages (dont 58 recueils du bulletin d'information de la Commission (Izvestija Tambovskoj učenoj arhivnoj komisssii - ITUAK) et 16 publications séparées). Le volume de son activité éditoriale la plaçait parmi les premières commissions d'archives russes - elle n'était devancée que par la commission de Tver'29.

La nomination de Norcov à la présidence de la Commission donna lieu à une nouvelle orientation de son activité, privilégiant l'étude des monuments de la culture matérielle. Les expéditions archéologiques sur le territoire de la province de Tambov commencèrent à y occuper une grande place. Pendant ces expéditions, on étudiait les objets du passé, on photographiait les vieilles églises et les vestiges des villes, on faisait des fouilles. La protection des monuments du passé devint une branche à part entière des activités de la Commission.

La révolution de 1905-1907 altéra le travail de la Commission, dont la situation financière se détériora considérablement à cause des interruptions survenues dans les cotisations des membres et les subventions des zemstva, dont les principaux payeurs - propriétaires fonciers et fonctionnaires - souffrirent énormément des troubles dans les campagnes. Les collaborateurs de la Commission d'archives cessèrent de participer aux congrès scientifiques de crainte que l'agitation qui s'était emparée de la société russe ne se reflétât sur leur travail et ne lui donnât un « air de partialité politique », ils limitèrent les contacts avec les autres sociétés historiques et se consacrèrent exclusivement à la recherche scientifique ${ }^{30}$. Cette ligne de conduite était inspirée par la position idéologique de la direction de la Commission et elle reflétait sa position sur les événements dans le pays et sur ce que devait être la place de l'homme de science en période d'agitation sociale. Norcov écrivit pendant ces années : «La science est un moyen unique et noble de s'éloigner de tous les extrêmes politiques, et même de les ignorer complètement et de réserver ainsi son cœur et son esprit pour le service de la patrie d'une façon pure

28. GATO,f. 178, op. 1, d. 66,1.4; d. 72,1.6.

29. V. P. Makarihin, « Gubernskie učenye arhivnye komissii i ih rol’ v razvitii obščestvennoistoričeskoj mysli v konce XIX-načale XX veka » (Les commissions scientifiques d'archives de gubernija et leur rôle dans le développement de la pensée socio-historique à la fin du XIX ${ }^{\mathrm{e}}$ siècle et au début du XX $\mathrm{X}^{\mathrm{e}}$ siècle), Istorija SSSR, 1, 1989, p. 167.

30. ITUAK, 52, 1906, p. 1 . 
et désintéressée. » ${ }^{31}$ Le chercheur désireux d'évaluer objectivement les événements historiques doit rester hors des partis et de la politique - telle était la conviction du président de la Commission.

L'apolitisme et le conservatisme dont fit preuve la Commission d'archives pendant la période révolutionnaire s'expliquent aussi par le fait que sa direction rejetait la lutte des classes, lui déniant la capacité de transformer la société. Très au fait de l'histoire de leur pays, les archivistes étaient convaincus que pour développer ce pays il fallait préférer la voie de l'évolution, que cette voie permettait de tenir suffisamment compte de l'expérience historique, de la mentalité et des réalisations culturelles du peuple russe. L'attitude du président de la Commission est également due au statut particulier de celle-ci au sein du système des institutions scientifiques. Grâce en grande partie aux efforts et aux relations personnelles de Norcov, la Commission de Tambov fut la première du groupe des commissions d'archives de province (GUAK) à être mise sous la protection du tsar en 1904.

Notons qu'aucun événement notable dans la vie de la ville n'eut lieu sans la participation de la Commission. Celle-ci joua un rôle important dans l'organisation de jubilées commémorant des événements locaux et nationaux, et fit même paraître dans ses Izvestija des essais et des articles sur les événements passés et présents les plus marquants du pays et de la région.

En 1912, la Commission exprima à la douma municipale son désir de renommer plusieurs des rues et squares de la ville qui ne portaient pas de noms historiques (Bol`̌saja, Gimnazičeskaja, Dolgaja... - la Grand'Rue, la rue du Lycée, la rue Longue...), en leur donnnant le nom de personnalités politiques et publiques qui avaient contribué au développement de Tambov: le gouverneur général de Tambov G. R. Deržavin; le voïvode fondateur de la ville Boborykin; le poète M. Ju. Lermontov, qui avait séjourné à Tambov et avait décrit la ville dans son récit Tambovskaja kaznačejša (La femme du caissier de Tambov); le gouverneur Gamaleja ; et E. D. Naryškin, qui avait fait don à la ville de l'Institut Alexandra, du Cercle équestre, du Cercle de la noblesse et d'une bibliothèque publique ${ }^{32}$. Cette initiative des membres de la Commission était en avance sur son temps. Actuellement, un tout petit nombre des personnalités proposées connaissent une seconde vie sur les plaques de rues et de squares de Tambov.

L'activité féconde et multiforme de la Commission a permis le développement de l'ethnographie historique dans la province de Tambov et a fortement contribué à la prise de conscience citoyenne des habitants de Tambov. C'est dans ce sens que ses présidents avaient œuvré - I. I. Dubasov, qui avait qualifié sa mission de «scientifique et patriotique » et A. N. Norcov, pour qui la Commission devait se consacrer à l'éducation de la génération actuelle et des générations futures, à l'éveil d'une «conscience nationale, d'un amour pour la patrie et d'une noble fierté nationale $»^{33}$.

31. Ibid., 53, 1909, p. 13.

32. Tambovskij kraj, 18 septembre 1912.

33. GATO, f. 4 , op. 1 , d. $5178,1.8 v^{\circ}$. 
L'intelligentsia a donc joué un rôle considérable dans les associations publiques de Tambov au tournant du $\mathrm{XX}^{\mathrm{e}}$ siècle. Le point de départ de son activité fut le désir d'élever le niveau et la qualité de vie des habitants de Tambov, de leur apporter civilisation et culture. Au cours de la création des organismes publics et pendant la durée de leur activité, l'intelligentsia dut faire face au dilemme russe essentiel de cette époque : quelle voie valait-il mieux adopter pour le développement de la Russie ? Celle des changements révolutionnaires radicaux ou celle de l'évolution et des «petites actions », qui nécessitait un travail méticuleux de construction? L'intelligentsia opta pour la seconde, celle de l'amélioration par étapes progressives de tous les aspects de la vie sans restructuration révolutionnaire douloureuse, dans le cadre d'organismes publics légaux.

Le caractère et les orientations de l'activité des associations publiques ont permis de changer la mentalité de la population de Tambov. En lui offrant la possibilité de se réunir et d'apporter une solution aux problèmes de sa vie quotidienne de façon autonome, les sociétés lui ont permis d'acquérir l'expérience de l'action citoyenne.

(traduit du russe par Christine Colpart)

Université d'État de Tambov

anastasiya13@mail.ru 